\title{
Bem-Estar Subjetivo e Traços de Personalidade em Crianças: Uma Relação Possível?
}

\author{
Letícia Garibaldi Gasparetto ${ }^{1}$ \\ Departamento de Desenvolvimento Humano, Universidade Federal do Rio Grande do Sul, \\ Porto Alegre, RS, Brasil \\ Cláudia Bandeira \\ Departamento de Psicologia, Universidade Federal do Rio Grande do Sul, \\ Porto Alegre, RS, Brasil \\ Claudia Hofheinz Giacomoni \\ Departamento de Psicologia, Centro Universitário La Salle, Canoas RS, Brasil
}

\begin{abstract}
Resumo
Este estudo teve como objetivo analisar as possíveis relações entre bem-estar subjetivo e traços de personalidade em 148 crianças brasileiras com idades entre cinco e 11 anos. As crianças eram estudantes do ensino básico e fundamental de duas escolas particulares e duas escolas da rede estadual do Rio Grande do Sul, Brasil. Os instrumentos utilizados foram a Escala Multidimensional de Satisfação de Vida para Crianças, a Escala de Afetos Positivos e Negativos para Crianças e a Escala de Traços de Personalidade para Crianças. Verificou-se que os afetos positivos correlacionaram positivamente com algumas dimensões da satisfação de vida como família e amizade, e com os traços extroversão e sociabilidade. Os afetos negativos apresentaram correlação positiva com os traços neuroticismo e psicoticismo. Esses achados são similares às correlações encontradas em adultos, bem como ampliam o entendimento da associação entre bem-estar subjetivo e traços de personalidade na infância.
\end{abstract}

Palavras-chave: Traços de personalidade, bem-estar subjetivo, criança.

\section{Subjective Well-Being and Personality Traits in Children: A Possible Relationship?}

\begin{abstract}
The aim of this study was to verify the relation of the subjective well-being and personality traits in 148 Brazilian children aged between five and 11 years. The children were primary and elementary schools students of Rio Grande do Sul, Brazil. The instruments used were the Multidimensional Scale of Life Satisfaction for Children, the Scale of Positive and Negative Affects for Children and the Scale of Personality Traits for Children. Results showed that positive emotions correlated positively with some dimensions of life satisfaction, as family and friends and with the traits of extroversion and sociability. Negative emotions were positively correlated with neuroticism traits and psychoticism. These findings are similar to correlations found in adults, as well as broaden the understanding between subjective wellbeing and personality traits in childhood.
\end{abstract}

Keywords: Personality traits, subjective well-being, children.

Endereço para correspondência: Rua Aureliano Figueiredo Pinto,483, Apto. 30, bairro: Praia de Belas, Porto Alegre, RS, Brasil. Fones: 51-32133398/51-81228829. E-mail: leticiagasparetto@yahoo.com.br 


\section{¿Bienestar Subjetivo y Rasgos de Personalidad en Niños: Una Relación Posible ¿}

\section{Resumen}

Este estudio investigó las relaciones entre bienestar subjetivo y rasgos de personalidad en 148 niños brasileños de entre cinco y 11 años. Los instrumentos utilizados fueron la Escala Multidimensional de Satisfacción con la Vida Infantil, Escala de Afectos Positivo y Negativo para la Infancia y Escala de Rasgos de la Personalidad para la Infancia. Se encontró que las emociones positivas se correlacionaron positivamente con las dimensiones de satisfacción con la vida como la familia y amigos y con los rasgos de la extraversión y la sociabilidad. Las emociones negativas se correlacionaron positivamente con los rasgos de neuroticismo y psicoticismo. Estos resultados son similares aquellos encontrados en los adultos. Expanden el entendimiento de la asociación de bienestar subjetivo y rasgos de personalidad en la infancia.

Palabras clave: Rasgos de personalidade, bienestar subjetivo, niño.

O bem-estar é uma preocupação antiga. Os primeiros estudos surgiram na filosofia com o objetivo de entender os aspectos inerentes à felicidade. Filósofos gregos como Platão, Sócrates e Aristóteles tinham como interesse a compreensão e a importância da felicidade na vida das pessoas. A experiência subjetiva tem sido estudada através de diferentes construtos, dentre eles o Bem-Estar Subjetivo (BES). O BES diz respeito a como e a por que as pessoas experienciam suas vidas de maneira positiva (Giacomoni, 2004). Este construto é formado por um fator global de variáveis interrelacionadas, constituído por componentes afetivos e cognitivos (Diener, Oishi, \& Lucas, 2003). O componente afetivo engloba as experiências de emoções prazerosas, os afetos positivos, e as experiências de emoções negativas, os afetos negativos. O componente cognitivo envolve o julgamento individual em relação à qualidade da vida (Bradshaw, Keung, Rees, \& Goswami, 2011). A avaliação cognitiva engloba a satisfação global com a vida e com componentes específicos como, por exemplo, escola, amizades, trabalho, religião (Giacomoni, 2002). Esta avaliação inclui uma análise pessoal sobre a frequência com que se experimentam emoções positivas e negativas.

Os Afetos Positivos e Negativos são compreendidos como a frequência, mais do que a intensidade com que as pessoas vivenciam emoções positivas ou negativas nas suas vidas (Lyu- bomirsky, King, \& Diener, 2005). Os Afetos Positivos dizem respeito à extensão do quanto uma pessoa está se sentindo entusiástica e alerta. Altos níveis de Afetos Positivos representam um estado de alta energia, total concentração e satisfação, enquanto baixos níveis de Afetos Positivos são caracterizados por tristeza e letargia. Por outro lado, Afetos Negativos é uma dimensão geral de insatisfação que inclui uma variedade de estados de humor aversivos, como raiva e angústia.

Os estudos do BES estão apoiados em teorias que buscam explicar o motivo de alguns indivíduos serem cronicamente felizes e satisfeitos com suas vidas (Steel, Schmidt, \& Shultz, 2008). Este construto tem sido fortemente correlacionado com traços estáveis da personalidade. Autores como Steel et al. (2008) acreditam que o estudo da personalidade é essencial para o entendimento do BES. As diferenças na personalidade e BES surgem cedo na vida e os componentes genéticos apresentam correlações moderadas a altas. As teorias que explicam a personalidade através do temperamento têm focado em três aspectos das diferenças individuais no BES. Em primeiro lugar, as pessoas apresentariam patamares únicos de BES que são determinados pela personalidade. Os outros aspectos residem nas diferenças de reatividade emocional e na forma como as pessoas processam as informações emocionais (Diener et al., 2003). 
Há concepções sustentando que traços de personalidade seriam responsáveis por regular as diferenças entre Afetos Positivos e Negativos (Wilson \& Gullone, 1999). A partir desta perspectiva, os níveis de afetos seriam sempre de similar magnitude ao longo do tempo, apesar do período de desenvolvimento do indivíduo, pois seriam determinados geneticamente. Há, contudo, outra concepção de entendimento da personalidade baseada nos afetos. Nela, cada pessoa nasce com níveis de afetos determinados que, combinados com as experiências de vida, produziriam traços de personalidade. Esses traços seriam responsáveis, da mesma forma, por regular os afetos, em um ciclo que se retroalimentaria. É uma concepção bidirecional, onde as emoções são tanto a causa quanto o efeito da personalidade (Yik \& Russell, 2001).

Estudos com adultos como o de Gilman e Huebner (2003) mostram a estabilidade e a consistência da Satisfação de Vida ao longo do tempo e de diferentes situações. Eles concluíram que escores de Extroversão e Neuroticismo (compreendidas como duas das cinco facetas que compõem a personalidade no modelo dos cinco grandes fatores) foram capazes de predizer o nível de Satisfação de Vida ao longo de um período de quatro anos. De acordo com esses autores, a avaliação da personalidade prediz, de forma mais consistente, a Satisfação de Vida do que os eventos da vida de uma pessoa.

Um estudo com gêmeos, realizado por Tellegen e colaboradores (1988), concluiu que há uma variação genética de aproximadamente $40 \%$ para Afetos Positivos, 55\% para Afetos Negativos e $48 \%$ de variação genética para Satisfação de Vida. Contudo, essas seriam propensões genéticas e significativas para crianças que se desenvolveram nas mesmas condições, já que a influência de diferentes fatores ambientais combinada com a genética é que seria significativa, na visão destes autores, para a constituição da personalidade.

\section{Personalidade}

O estudo da personalidade é embasado em teorias que englobam diferentes esferas compor- tamentais e cognitivas. Algumas contribuições recentes neste campo compreendem a personalidade como um conjunto de características do indivíduo que justificam estilos persistentes na forma de sentir, pensar e se comportar diante dos eventos (Löckenhoff, Terracciano, Patriciu, Eaton, \& Costa, 2009). Outros autores sugerem o entendimento da personalidade como aquilo que se refere à singularidade, consistência e estabilidade do comportamento (Sisto, 2004). O constituinte biológico da personalidade é conhecido como temperamento. A abordagem de Rothbart (2004) define temperamento como diferenças individuais com base constitucional na reatividade e auto-regulação, observadas nos domínios de emocionalidade, atividade motora e atenção, sendo influenciados pela hereditariedade, maturação e experiência.

Cattel e Allport são considerados grandes estudiosos da personalidade. Para Cattel, a personalidade pode ser entendida como a capacidade de predizer o que uma pessoa faria em uma determinada situação. Para Allport, a unidade básica da personalidade é o traço, sendo que a enumeração dos traços de uma pessoa forneceria uma descrição de sua personalidade. Os traços poderiam ser inferidos além da linguagem, a partir dos comportamentos manifestos das pessoas e até mesmo de documentos e registros de vida das mesmas (Schultz, 2011).

Embora desse continuidade ao modelo de análise fatorial de Cattell, a abordagem de Hans J. Eysenck focou em uma cadeia causal em que um substrato biológico é responsável pelas diferenças individuais em dimensões fundamentais da personalidade. Para Eysenck (1967, 1997), um modelo adequado da personalidade precisaria incluir uma taxonomia descritiva e especificar mecanismos biológicos que contribuam para as diferenças observadas nessas dimensões descritivas. O modelo de Eysenck da personalidade inclui três dimensões tipológicas básicas: introversão versus Extroversão, Neuroticismo versus estabilidade e Psicoticismo versus controle de impulsos (Eysenck, 1967, 1997). O traço Extroversão poderia ser considerado a dimensão responsável pela impulsividade. A pessoa com alta pontuação nesse traço pode ser descrita como 
impulsiva, despreocupada, agressiva, em busca de sensações, otimista, espontânea e aberta às relações interpessoais. Normalmente é dominante, aventureira, sociável, assertiva, vivaz e animada. As pessoas extrovertidas têm muitos amigos e gostam de conversar. São sujeitos que normalmente arriscam e que gostam de mudanças (Eysenck, 1967, 1997; Sisto, 2004).

O traço de personalidade Neuroticismo estaria relacionado à reatividade do sistema nervoso autônomo. Como componentes do Neuroticismo podem ser citados ansiedade, depressão, sentimento de culpa, baixa autoestima, timidez, melancolia, tristeza, temor, nervosismo, inquietação e emotividade. A principal característica dessas pessoas é uma constante preocupação, bem como instabilidade emocional.

Por fim, altas pontuações em Psicoticismo é indicativo de inflexibilidade, bem como indica pessoas que têm uma certa despreocupação com relação aos outros, com tendência a serem cruéis, desumanas e insensíveis, mostrando-se hostis, até mesmo com os mais íntimos. São normalmente egocêntricas, impessoais, não-empáticas, frias e agressivas, podendo ser criativas (Sisto, 2004).

$\mathrm{O}$ questionário de Eysenck se detém em aspectos comportamentais e não da constituição da personalidade. Caracterizam, por isso, tendências com possibilidade de mudança, pois consideram as interações entre o constituinte biológico da pessoa e os constituintes do meio como potenciais promotores dessas mudanças.

Atualmente, a compreensão de que a personalidade se dá através de transações recíprocas entre o indivíduo e os fatores ambientais vem crescendo (Kiff, Lengua, \& Zalewsky, 2011). Além disso, a pesquisa feita por Abe (2005), por exemplo, com uma amostra de 59 mães e seus bebês, mostra que crianças que foram avaliadas pelas mães aos três anos e meio, mais tarde, aos cinco anos por experimentadores em sala de espelhos e posteriormente, aos 12 anos demonstraram poucas modificações em suas personalidades com o decorrer do tempo.

A tarefa de extrair auto-relatos confiáveis e válidos de crianças pequenas pode ser complicada por fatores que incluem suas habilidades cognitivas limitadas, seus níveis de engajamen- to com o entrevistador ou com os métodos de auto-relato e a tendência de a criança enviesar as respostas para que sejam sempre positivas (Measelle, John, Ablow, Philip, \& Carolyn, 2005). Contudo, sabe-se que a idade de cinco a sete anos é um importante período do desenvolvimento durante o qual maiores mudanças cognitivas têm sido vinculadas com mudanças na capacidade auto-representacional, o que facilitaria o processo avaliativo das crianças (Measelle et al., 2005).

Estudos com crianças têm sido considerados controversos devido à avaliação das crianças ser feita por terceiros e não por elas mesmas. Measelle e colaboradores (2005) evidenciaram em suas pesquisas como as crianças, mesmo muito novas, já conseguem falar sobre aspectos de sua personalidade. Neste estudo, crianças de cinco anos conseguiram falar relativamente bem sobre aspectos de sua personalidade, enquanto crianças de seis e sete anos apresentaram respostas coerentes, equivalentes às respostas dos adultos às mesmas questões. $\mathrm{O}$ estudo mostrou que crianças aos seis anos de idade conseguem perceber melhor seus sentimentos de tristeza e ansiedade do que seus pais e professores.

A importância do estudo com crianças se dá por pelo menos duas razões principais: a primeira é o fato de que o bem-estar infantil não é restrito à vida presente delas, tendo repercussões futuras. A segunda é que há ainda poucas pesquisas realizadas diretamente com crianças (Fernandes, Mendes, \& Teixeira, 2012).

Considerando as relações entre bem-estar subjetivo e traços de personalidade, sabe-se que fatores de personalidade têm se destacado como possíveis indicadores de bem-estar subjetivo (BES; Steel et al., 2008; Zanon, Bastianello, Pacico, \& Hutz, 2013), juntamente com fatores objetivos como o ciclo da vida do sujeito (pois os objetivos e o entendimento dos acontecimentos da vida de um sujeito vão mudando com o seu amadurecimento, influenciando na percepção de bem-estar) bem como com a cultura e o nível socioeconômico no qual ele está inserido (Bradshaw et al., 2011). Devido a estes fatores contextuais e temporais influenciarem no bem-estar subjetivo, a presente pesquisa foi realizada com 
crianças da mesma faixa etária e mesma cultura, avaliando sua Satisfação de Vida, afetos negativos e positivos e a relação destes com traços de personalidade.

\section{Método}

\section{Participantes}

Participaram do estudo 148 crianças, 50,7\% do sexo masculino, com idades entre cinco e 11 anos $(M=8,17, D P=1,80)$ de uma escola privada do interior do estado do Rio Grande do Sul. No que se refere à posição da criança na família, $39,9 \%$ eram os filhos mais novos e $27,7 \%$ os filhos mais velhos.

\section{Instrumentos}

Foi utilizada uma ficha de identificação com questões sociodemográficas como idade, sexo, escola, série, número de irmãos, com o objetivo de caracterizar a amostra. Além deste foram utilizados outros instrumentos:

Escala de Afetos Positivos e Negativos para Crianças - Pré-escola (Giacomoni, Bandeira, \& Hutz, 2017). A Escala de Afetos Positivos e Negativo para Crianças- Pré-escola é composta por 30 itens, sendo 15 itens da subescala de Afetos Positivos e 15 itens da subscala de Afetos Negativos. É utilizada uma escala de respostas do tipo Likert de cinco pontos, sendo as possibilidades de resposta: (1) nem um pouco; (2) um pouco; (3) mais ou menos; (4) bastante; (5) muitíssimo. O coeficiente alfa da escala total, nos estudos de construção da escala, foi de $\alpha=0,90$. Para a subescala de Afetos Positivos o índice foi $\alpha=0,88$ e para a subescala de Afetos Negativos foi de $\alpha=0,84$. Originalmente elaborada para ser respondida por crianças de sete a 12 anos, a escala foi adaptada por Giacomoni, Bandeira e Hutz de forma que os itens foram lidos e foi criada uma escala pictórica de quatro pontos onde as crianças apontavam o quadrado correspondente à resposta escolhida: "concordo totalmente" (quadrado completamente cheio), "concordo" (quadrado parcialmente cheio), "discordo" (quadrado parcialmente vazio) e "discordo totalmente" (quadrado totalmente vazio).
Escala Multidimensional Satisfação de Vida para Crianças-Versão Reduzida (Giacomoni, Bandeira, Zanon, \& Hutz, 2015). A Escala Multidimensional de Satisfação de Vida para Crianças-Versão Reduzida é uma medida multidimensional cujo objetivo é avaliar a Satisfação de Vida das crianças a partir de domínios específicos de suas vidas. $\mathrm{O}$ estudo original apontou consistência interna satisfatória $(\alpha=0,93)$. A escala na sua versão reduzida possui 27 itens divididos entre quatro fatores: Self Comparado, Família, Amizade e Escola. Para responder às questões, a criança seleciona uma das cinco opções de frequência: (1) nem um pouco, (2) um pouco, (3) mais ou menos, (4) bastante, (5) muitíssimo. Também essa escala foi adaptada para a utilização em crianças menores de oito anos (Giacomoni et al., 2015) e seguiu os mesmos procedimentos utilizados na Escala de Afetos Positivos e Negativos para Crianças.

Escala de Traços de Personalidade para Crianças- ETPC (Sisto, 2004). A ETPC está configurada para ser respondida por crianças de cinco a 10 anos, sem histórico conhecido de patologia psicológica. O instrumento possui um total de 30 itens com opção de resposta dicotômica e apresentou consistência interna superior a 0,80 . A consistência interna para psicoticismo foi de 0,91 , para extroversão e sociabilidade 0,88 e para neuroticismo, 0,80 (Sisto, 2004). A escala apresenta validade de constructo e de conteúdo para medir quatro fatores originados da teoria dos traços de personalidade de Eysenck: Neuroticismo, Psicoticismo, Extroversão e Sociabilidade.

\section{Procedimentos Éticos}

Foi solicitada a concordância da escola participante através de um documento escrito de forma a realizar o estudo. Os pais ou responsáveis pelas crianças foram contatados através de um documento escrito, com esclarecimentos sobre a pesquisa. Salvaguardou-se a todos os participantes o direito de sigilo, voluntariado e interrupção da participação. O estudo está de acordo com a Resolução $n^{\circ}$. 466/2012 do Conselho Nacional de Saúde, que versa sobre os aspectos éticos da pesquisa com seres humanos. Todos os procedi- 
mentos atenderam as recomendações do Comitê de Ética e Pesquisa do Instituto de Psicologia da Universidade Federal do Rio Grande do Sul (UFRGS), o qual aprovou o estudo sob o protocolo n ${ }^{\circ} 22623$.

\section{Procedimentos de Coleta}

A coleta de dados foi realizada nas dependências das escolas, em salas de aula destinadas à realização da pesquisa. Os instrumentos foram aplicados por uma equipe de psicólogos e graduandos de Psicologia previamente treinados. As crianças menores de nove anos responderam individualmente, enquanto os demais responderam de forma coletiva.

Para as crianças menores de nove anos, a aplicação foi individual, com instrumentos compatíveis à sua faixa etária e etapa de desenvolvimento. Esses instrumentos apresentam versões adaptadas e/ou criadas para este fim. Os itens de todos os instrumentos foram lidos para as crianças e suas respostas foram auxiliadas por escalas Likert pictóricas (Giacomoni et al., 2017), com quadrados preenchidos completamente, parcialmente, ou vazios correspondente à resposta escolhida. A aplicação a partir deste método foi bastante eficaz, havendo pouca ou nenhuma dificuldade por parte das crianças.

Todos os alunos receberam informações sobre os objetivos do estudo através de uma linguagem apropriada para cada faixa etária. Foi dito aos participantes que gostaríamos de saber o que pensam a respeito de algumas coisas que fazem parte de suas vidas, e de como se sentem a respeito. Todos foram informados verbalmente que sua participação era voluntária e que poderiam interrompê-la a qualquer momento sem nenhuma penalidade. Foram prestadas informações quanto ao sigilo e à confidencialidade dos dados coletados. Os participantes foram encorajados a responder honestamente e informados que não existiam respostas certas ou erradas. A coleta de dados foi realizada em uma única sessão com tempo aproximado de 45 minutos.

\section{Procedimentos de Análise}

Com o objetivo de verificar possíveis correlações entre as variáveis estudadas, bem-estar subjetivo e traços de personalidade em crianças, realizou-se como método de análise a correlação de Pearson, que mede o grau e a direção da correlação entre variáveis. Assim, foi possível verificar a interdependência das variáveis estudadas.

\section{Resultados}

O objetivo do estudo foi analisar as possíveis relações entre bem-estar subjetivo e traços de personalidade em crianças com idades entre cinco e 11 anos, utilizando a Escala Multidimensional de Satisfação de Vida para Crianças, a Escala de Afetos Positivos e Negativos para Crianças e a Escala de Traços de Personalidade para Crianças (ETPC). Inicialmente foram realizadas análises descritivas, apresentadas na Tabela 1.

Tabela 1

Propriedades da Escala de Afetos Positivos e Negativos, da Escala Multidimensional de Satisfação de Vida Infantil e da ETPC

\begin{tabular}{lccc}
\hline Fatores & Itens & Média & $D P$ \\
\hline Afetos Positivos & 15 & 4,30 & 0,48 \\
Afetos Negativos & 15 & 1,70 & 0,69 \\
Self Comparado & 6 & 3,69 & 0,88 \\
Família & 8 & 4,78 & 0,29 \\
Amizade & 7 & 4,69 & 0,39 \\
Escola & 6 & 4,63 & 0,54 \\
Psicoticismo & 11 & 1,95 & 1,36 \\
Extroversão & 10 & 6,94 & 1,16 \\
Neuroticismo & 7 & 3,51 & 1,76 \\
Sociabilidade & 6 & 4,22 & 1,43 \\
\hline
\end{tabular}


Tabela 2

Correlações entre Afetos Positivos e Negativos e Fatores de Satisfação de Vida

\begin{tabular}{lcccccc}
\hline & Família & Amizade & Escola & SelfComparado & $\begin{array}{c}\text { Afetos } \\
\text { Positivos }\end{array}$ & $\begin{array}{c}\text { Afetos } \\
\text { Negativos }\end{array}$ \\
\hline Família & & & & & & \\
Amizade & $0,51^{* *}$ & & & & & \\
Escola & $0,34^{* *}$ & $0,45^{* *}$ & & & & \\
Self Comparado & $0,21^{*}$ & $0,25^{* *}$ & $0,18^{*}$ & & & \\
Afetos Positivos & $0,36^{* *}$ & $0,54^{* *}$ & $0,33^{* *}$ & 0,06 & & \\
Afetos Negativos & $-0,12$ & $-0,26^{* *}$ & $-0,06$ & $-0,35^{* *}$ & $-0,18^{*}$ & \\
Satisfação Total & $0,58^{* *}$ & $0,68^{* *}$ & $0,66^{* *}$ & $0,77^{* *}$ & $0,37^{* *}$ & $-0,32^{* *}$ \\
\hline
\end{tabular}

Notas. ** Correlação é significativa no nível 0,01 (2-tailed). * Correlação é significativa no nível 0,05 (2-tailed).

$\mathrm{Na}$ Tabela 2, foram verificadas as correlações entre as escalas de Afetos Positivos e Negativos e a Escala Multidimensional de Satisfação de Vida Infantil.

Observa-se que as maiores correlações de Afetos Positivos estão relacionadas à Amizade $(r=0,54, p<0,01)$, Satisfação Total $(r=0,37, p<$ $0,01)$, Família $(r=0,36, p<0,01)$ e Escola $(r=$ $0,33, p<0,01)$, o que sugere que as relações interpessoais são fortes indicativos de Afetos Positivos, corroborando com estudos anteriores (Bradshaw et al., 2011; Giacomoni, 2002).

Tabela 3

Traços de Personalidade, Afetos Positivos e Negativos e Satisfação de Vida

\begin{tabular}{lcccc}
\hline & Psicoticismo & Extroversão & Neuroticismo & Sociabilidade \\
\hline Afetos Positivos & $-0,29^{* *}$ & $0,30^{* *}$ & $-0,06$ & $0,31^{* *}$ \\
Afetos Negativos & $-0,21^{*}$ & 0,04 & $0,25^{* *}$ & $-0,41^{* *}$ \\
Família & $-0,21^{*}$ & $0,18^{*}$ & $-0,08$ & $0,18^{*}$ \\
Amizade & $-0,31^{* *}$ & 0,15 & $-0,25^{* *}$ & $0,33^{* *}$ \\
Escola & $-0,15$ & 0,06 & $-0,11$ & $0,22^{* *}$ \\
Self-Comparado & $-0,24^{* *}$ & 0,00 & $-0,27^{* *}$ & $0,18^{*}$ \\
Satisfação Total & $-0,33^{* *}$ & 0,10 & $-0,29^{* *}$ & $0,32^{* *}$ \\
\hline
\end{tabular}

Notas. ** Correlação é significativa no nível 0,01 (2-tailed). * Correlação é significativa no nível 0,05 (2-tailed).

Conforme a Tabela 3, os Afetos Positivos foram correlacionados com o traço de personalidade Sociabilidade $(r=0,31, p<0,01)$ e Extroversão $(r=0,30, p<0,01)$, o que corrobora com achados anteriores de adultos. Afetos Positivos tiveram correlação com o traço de personalidade Psicoticismo $(r=-0,29, p<0,01)$, dado que necessita ser melhor investigado.
Afetos Positivos apresentaram uma correlação de .06 com Neuroticismo, o que corrobora com estudos anteriores (Nunes, Giacomoni, \& Hutz, 2009). Afetos Negativos apresentaram correlação com traço de personalidade Sociabilidade $(r=0,41, p<0,01)$ e uma baixa correlação com traço de personalidade Extroversão ( $r=$ $0,04, p<0,01)$. Os Afetos Negativos também 
estiveram positivamente correlacionados ao fator Psicoticismo $(r=0,42, p<0,01)$.

As correlações entre Afetos Negativos e Neuroticismo foram $r=0,25(p<0,01)$. Satisfação de Vida Total apresentou correlações significativamente medianas com Neuroticismo e Psicoticismo (respectivamente $r=-0,29(p<0,01) \mathrm{e}$ $r=-0,33(p<0,01)$. Quase todos os fatores da Satisfação de Vida apresentaram correlação significativa com o traço de personalidade Sociabilidade: Amizade $(r=0,33, p<0,01)$, Escola $(r=0,22$, $p<0,01)$ e Satisfação Total $(r=0,32, p<0,01)$. Satisfação de Vida apresentou correlações significativas com Self Comparado ( $r=0,77, p<0,01)$, Amizade $(r=0,68, p<0,01)$, Escola $(r=0,66$, $p<0,01)$ e Família $(r=0,58, p<0,01)$.

\section{Discussão}

Sabendo que os Afetos Negativos são traduzidos por um conjunto de emoções negativas (Giacomoni, 2002), esta correlação veio ao encontro de outros estudos que avaliaram traços de personalidade e emoções. Sisto (2004) avaliou traços de personalidade e emoções em crianças de oito a 10 anos de idade e os resultados demonstraram a associação entre Psicoticismo e uma baixa pontuação em alegria e alta pontuação em tristeza.

Estudos com adultos baseados no Big Five demonstraram que Afetos Negativos estão negativamente associados com Extroversão (Nunes et al., 2009). O Big Five desenvolveu-se a partir das pesquisas realizadas na área das teorias fatoriais e das teorias de traços de personalidade, as últimas contribuindo grandemente para o desenvolvimento da sua base teórica. Já as teorias fatoriais contribuíram sob o aspecto instrumental e metodológico. Esse processo deu-se também a partir do avanço das tecnologias da computação, além da elaboração de métodos mais sofisticados de localização e extração de fatores, que acabaram dando respaldo a essa forma de organização e explicação da personalidade (Nunes, Hutz, \& Nunes, 2010). Portanto, podemos pensar a partir disso que crianças extrovertidas e interessadas no bem-estar dos outros parecem vivenciar bai- xos níveis de Afetos Negativos. Segundo Löckenhoff e colaboradores (2009), pessoas com altos escores em Neuroticismo percebem mais eventos negativos, enquanto pessoas extrovertidas e sociáveis fariam o contrário.

Contudo, há uma diferença nestas associações quando os estudos são com crianças.

O estudo de Wilson e Gullone (1999) relacionou os traços de personalidade Neuroticismo e Extroversão respectivamente com Afetos Negativos e com Afetos Positivos e percebeu que estas relações existiam, embora fossem de fracas a moderadas em crianças e adolescentes, explicando respectivamente 10 e 18\% da variância compartilhada. Em adultos, estas relações se mostraram mais fortes. Estes achados corroboram a perspectiva de que personalidade e afetos se tornam mais fortemente relacionados com o aumento da idade.

As correlações entre Afetos Negativos e Neuroticismo replicaram estudos documentados na literatura com relação a adultos (Abe, 2005; Gomez, Krings, Bangerter, \& Grob, 2009; Nunes et al., 2009; Zanon, 2011). Já Extroversão e Afetos Positivos apresentaram considerável correlação pelo fato de sujeitos extrovertidos serem sociáveis, assertivos, enérgicos e ativos (Zanon et al., 2013). Extroversão e Sociabilidade estão relacionadas a vivências interpessoais e afetos positivos.

Satisfação de Vida Total apresentou correlações significativamente medianas com Neuroticismo e Psicoticismo. Crianças com esse perfil tenderiam a apresentar uma avaliação mais negativa sobre si mesmas, bem como vivenciariam altos níveis de sofrimento psicológico, solidão e uma percepção de que os eventos de sua vida são incontroláveis (lócus de controle externo). Esses indicativos são relevantes, já que estudos epidemiológicos indicam que cerca de uma em cada cinco crianças apresenta alguma questão psicológica ao longo da infância.

Bradshaw e colaboradores (2011) apontam em seu estudo que a Satisfação de Vida em crianças e pré-adolescentes está relacionada principalmente ao relacionamento da criança com os pares. Características pessoais como lócus de 
controle interno, autoestima, autoconfiança e autoeficácia também têm sido consistentemente associadas com Satisfação de Vida. Corroborando com estes estudos, quase todos os fatores da Satisfação de Vida apresentaram correlação significativa com o traço de personalidade Sociabilidade. A escala de Sociabilidade informa sobre a tendência a comportar-se dentro de regras de convivência sociais. Portanto, é possível concluir que a Sociabilidade se dá expressivamente nos contextos onde essas correlações foram significativas para as crianças, como na escola, onde se encontrou a correlação mais alta. Satisfação de Vida apresentou correlações significativas com Self Comparado, Amizade, Escola e Família. Essas correlações também apareceram como significativas em estudos como o de Giacomoni (2002).

Atualmente, não há duvidas de que a fase inicial da vida tem repercussão na constituição física, emocional e cognitiva dos sujeitos (Piccoloto \& Wainer, 2007). Devido à evolução das características de alguns transtornos ao longo da infância e adolescência (Fundo das Nações Unidas para a Infância [UNICEF], 2009), passouse a enfatizar a relevância clínica e diagnóstica dos problemas comportamentais de crianças com menos de seis anos como indicadores de possíveis dificuldades futuras. Contudo, infelizmente a área da saúde mental infantil ainda recebe pouca atenção de pesquisadores e clínicos (Caminha \& Caminha, 2007). O estudo da Satisfação de Vida, afetos positivos e negativos e sua relação com traços de personalidade pode contribuir para a compreensão, explicação e possível predição da manifestação do bemestar subjetivo. Os achados do presente estudo com crianças corroboraram as associações já conhecidas e realizadas por meio de estudos de análise fatorial entre personalidade e bem-estar subjetivo em adultos. Embora as evidências sugiram que várias medidas de Satisfação de Vida em crianças e adolescentes mostram substancial validade com crianças acima de oito anos (Gilman, Huebner, \& Laughlin, 2000), foi possível apreender a partir deste estudo a percepção de crianças ainda menores sobre o seu próprio bem-estar subjetivo.
Tais estudos serão úteis no desenvolvimento de intervenções eficientes, dado que a criança ainda está se desenvolvendo e consequentemente está mais vulnerável a intervenções do meio. Essas intervenções, realizadas nas escolas, como oficinas de mindfulness para crianças que apresentam altos escores de Neuroticismo, por exemplo, poderiam permitir o aumento dos níveis de bem-estar e, consequentemente, da qualidade de vida dessas crianças quando adultas.

Com base nos achados descritos anteriormente, a importância deste trabalho se dá pelo incentivo que ele fornece a novas investigações que verificariam se as relações entre as variáveis apresentadas no estudo citado são recorrentes. Como a Psicologia Positiva no Brasil ainda está em fase inicial, muitos estudos focados nas questões levantadas neste trabalho são verificados em sua grande maioria apenas na literatura internacional. Espera-se que este estudo possa ser apenas o início de posteriores investigações brasileiras entre bem-estar subjetivo e traços de personalidade em crianças.

\section{Referências}

Abe, J. A. A. (2005). The predictive validity of the Five-Factor Model of personality with preschool age children: A nine year follow-up study. Journal of Research in Personality, 39(4), 423-442. doi:10.1016/j.jrp.2004.05.002

Bradshaw, J., Keung, A., Rees, G., \& Goswami, H. (2011). Children's subjective well-being: International comparative perspectives. Children and Youth Services Review, 33(4), 548-556. doi:10.1016/j.childyouth.2010.05.010

Caminha, R. M., \& Caminha, M. G. (2007). A prática cognitiva na infância. São Paulo, SP: Roca.

Diener, E., Oishi, S., \& Lucas, R. E. (2003). Personality, culture, and subjective well-being: Emotional and cognitive evaluations of life. Annual Review of Psychology, 54, 403-425. doi:10.1146/ annurev.psych.54.101601.145056

Eysenck, H. J. (1967). The biological basis of personality. Springfield, IL: Charles C. Thomas.

Eysenck, H. J. (1997). Personality and experimental psychology: The unification of psychology and the possibility of a paradigm. Journal of Personality and Social Psychology, 73, 1224-1237. 
Fernandes, L., Mendes, A., \& Teixeira, A. A. C. (2012). Review essay on the measurement of child well-being. Social Indicators Research, 106(2), 239-257. doi:10.1007/s11205-0119814-9

Fundo das Nações Unidas para a Infância. (2009). The State of the world's children 2009. Retrieved from http://www.unicef.org/sowc09/ docs/SOWC09-FullReport-EN.pdf

Giacomoni, C. H. (2002). Bem-estar subjetivo infantil: Conceito de felicidade e construção de instrumentos para avaliação (Tese de doutorado, Curso de Pós-Graduação em Psicologia do Desenvolvimento, Universidade Federal do Rio Grande do Sul, Porto Alegre, RS, Brasil).

Giacomoni, C. H. (2004). Bem-estar subjetivo: Em busca da qualidade de vida. Temas em Psicologia da SBP, 12(1), 43-50.

Giacomoni, C. H., Bandeira, C., \& Hutz, C. S. (2017). Escala de Afetos Positivos e Negativos para crianças a partir de 5 anos. Manuscrito em preparação.

Giacomoni, C. H., Bandeira, C., Zanon, C., \& Hutz, C. (2015). Escala Multidimensional de Satisfação de Vida para Crianças - Versão Reduzida. Manuscrito em preparação.

Gilman, R., \& Huebner, S. (2003). A review of Life Satisfaction Research with Children and Adolescents. School Psychology Quarterly, 18(2), 192 205. doi:10.1521/scpq.18.2.192.21858

Gilman, R., Huebner, E. S., \& Laughlin, J. E. (2000). A first study of the Multidimensional Students' Life Satisfaction Scale with adolescents. Social Indicators Research, 52(2), 135-160. doi:10.1023/A:1007059227507

Gomez, V., Krings, F., Bangerter, A., \& Grob, A. (2009). The influence of personality and life events on subjective well-being from a life span perspective. Journal of Research in Personality, 43, 345-354. doi:10.1016/j.jrp.2008.12.014

Kiff, C. J., Lengua, L. J., \& Zalewski, M. (2011). Nature and nurturing: Parenting in the context of child temperament. Clinical Child and Family Psychology Review, 14(3), 251-301. doi: 10.1007/s10567-011-0093-4.

Lyubomirsky, S., King, L., \& Diener, E. (2005). The benefits of frequent positive affect: Does happiness lead to success. Psychological Bulletin, 131(6), 803-855. doi:10.1037/00332909.131.6.803
Löckenhoff, C. E., Terracciano, A., Patriciu, N. S., Eaton, W. W., \& Costa P. T., Jr. (2009). Self-reported extremely adverse life events and longitudinal changes in five-factor modelpersonality traits in an urban sample. Journal of Traumatic Stress, 22(1), 53-59. doi:10.1002/jts.20385

Measelle, J. R., John, O. P., Ablow, J. C., Philip A. C., \& Carolyn, P. C. (2005). Can children provide coherent, stable, and valid self-reports on the bigfive dimensions? A longitudinal study from ages 5 to 7. Journal of Personality and Social Psychology, 89(1), 90-106. doi:10.1037/00223514.89.1.90

Nunes, C. H. S., Giacomoni, C. H., \& Hutz, C. S. (2009). Associação entre Bem-Estar Subjetivo e personalidade no modelo dos Cinco Grandes Fatores. Avaliação Psicológica, 8(1), 99-108.

Nunes, C. H. S. S., Hutz, C. S., \& Nunes, M. F. O. (2010). Bateria Fatorial de Personalidade (BFP): Manual técnico. São Paulo, SP: Casa do Psicólogo.

Piccoloto, N. M., \& Wainer, R. (2007). Aspectos biológicos da estruturação da personalidade e terapia cognitivo-comportamental. In R. M. Caminha \& M. G. Caminha (Eds.), A prática cognitiva na infância (pp. 16-35). São Paulo, SP: Rocca.

Rothbart, M. K. (2004). Commentary: Differentiated measures of temperament and multiple pathways to childhood disorders. Journal of Clinical Child and Adolescence Psychology, 33(1), 8287. doi:10.1207/S15374424JCCP3301_8

Schultz, D. P. (2011). Teorias da personalidade (2. ed.). São Paulo, SP: Cengage Learning.

Sisto, F. F. (2004). Escala de Traços de Personalidade para Crianças. São Paulo, SP: Vetor.

Steel, P., Schmidt, J., \& Schultz, J. (2008). Refining the relationship between personality and subjective well-being. Psychological Bulletin, 134(1), 138-161.

Tellegen, A., Lykken, D. T., Bouchard, T. J., Jr., Wilcox, K., Segal, N., \& Rich, S. (1988). Personality similarity in twins reared apart and together". Journal of Personality and Social Psychology, 54(6), 1031-1039.

Wilson, K., \& Gullone, E. (1999). The relationship between personality and affect over the lifespan. Personality and Individual Differences, 27, 1141-1156. 
Yik, M. S. M., \&. Russell, J. A. (2001) Predicting the Big Two of Affect from the Big Five of Personality. Journal of Research in Personality, 35(3), 247-277. doi:10.1006/jrpe.2001.2322

Zanon, C. (2011). Construção de uma escala de afetos e suas relações com os cinco grandes fatores da personalidade (Big Five) (Tese de doutorado, Curso de Pós-Graduação em Psicologia do Desenvolvimento, Universidade Federal do Rio Grande do Sul, Porto Alegre, RS, Brasil).
Zanon, C., Bastianello, M. R., Pacico, J. C., \& Hutz, C. S. (2013). Relações entre Afetos Positivos e Negativos com os Cinco Fatores da Personalidade em uma amostra brasileira. Paidéia (Ribeirão Preto), 23(56), 285-292. doi:10.1590/198243272356201302

Recebido: $21 / 07 / 2015$

$1^{a}$ revisão: $20 / 01 / 2016$ Aceite final: 07/03/2016 